\title{
Treatment of Diabetic Foot Ulcer With An Epidemiological Approach: A systematic Review Study
}

\author{
Zahra Shiri' ${ }^{1}$ Zeinab Zarinzade ${ }^{1}$, Mohammad Khalilikia², Ahmad Aslani², Hadi \\ Kianirad $^{*}$, Yones Adish ${ }^{2}$ \\ ${ }^{1}$ Student Research Committee, Ahvaz Jundishapur University of Medical Sciences, Ahvaz, Iran. \\ ${ }^{2}$ Student Research Committee, Dezful University of Medical Sciences, Dezful, Iran.
}

\begin{abstract}
Introduction: Diabetes is a very dangerous disease in the new age, and foot ulcer is one of its complications. If the foot ulcer is not quickly and properly treated, it may lead to amputation. Therefore, due to the importance of this issue, this systematic review was conducted with the aim of determining the treatment of diabetic foot ulcer with an epidemiological approach.
\end{abstract}

Materials and Methods: The present study is a systematic review study that sought scientific articles in search engines, sites, and databases. This study, using the articles published over the past 24 years, has been associated with effective treatments for the accelerated healing of diabetic foot ulcers. In the first phase, 36 articles were found. of these, 12 articles related to the topic that was published in the last 24 years were reviewed.

Results: In this study, several methods for the treatment of diabetic foot ulcers have been investigated. One of these methods that work in the treatment of foot ulcers is the "air cast diabetic walker boot" that inflates the airbag inside the boot to reduce stress on the skin. It has a hard outer cover and a deep heel that lowers the pressure.

Conclusion: In the studies that have been studied, several therapeutic methods have been used to diabetic foot ulcers that have been effective. Considering that serious measures have been taken around the world to reduce complications of diabetes, including foot ulcers, but diabetes research centers in various countries and researchers working on diabetes must have serious plans to reduce complications of diabetic foot ulcers design and implement.

Keywords: Foot ulcer, Diabetic patients, Diabetic foot ulcer, Epidemiologic approach, Diabetic foot ulcer treatment.

\section{Introduction}

Diabetes mellitus is considered as the most common disease caused by metabolic disorders and is a major global challenge. It is a disorder of insulin production and function disorder, which has led to an increase in uncontrolled outbreaks due to decreased physical activity and the consumption of unhealthy foods. (1-9). In 2012, the direct cause of diabetes was 1.5 million deaths, with an inappropriate combination (low physical activity and unhealthy diet) leading to an uncontrollable increase in the prevalence of diabetes in the world, and in 2014, the global prevalence of diabetes among adults over the age of 18 The year was estimated at $9 \%$. (19-19) and the prevalence of this disease in Iran, according to the statistics released by the health department of the Ministry of Health in a population over the age of 30, is more than $14 \%$ or more in the female population, and it is associated with multiple short-term and long-term complications, which in many 
cases is not reversible and there are many complications such as blindness, neuropathy and cardiovascular discomfort (20-38).

In diabetic patients, depression is one of the most common psychiatric disorders that depression is a mood that involves rage and escapes from activity or unconsciousness and reluctance, and can affect one's thoughts, behavior, feelings and health, and well-being. Depression is one of the most common and debilitating problems for youth and adolescents. And depression and occupational stress may cause some disorders in the mental and physical health of individuals, and high occupational stress is known as a known psychosocial factor in the development of cardiovascular disease (39-45). And diabetes is one of the most common endocrine complications in thalassemic patients, and thalassemia is a hereditary condition of the disease and occurs in both alpha thalassemia and beta thalassemia. Beta-thalassemia is a group of hereditary blood disorders caused by the reduction of synthesis of beta-hemoglobin in the blood chain. The annual incidence of symptomatic cases of this disease is estimated at one in 100,000 worldwide (46-54).

A foot ulcer is a major complication of diabetes mellitus with high morbidity, mortality, and associated costs, and therefore necessitates precautionary measures against diabetes mellitus (9). Therefore, due to the importance of this issue, this systematic review was conducted with the aim of determining the treatment of diabetic foot ulcer with an epidemiological approach.

\section{Materials and Methods}

In order to achieve the goal of the study and to improve the accuracy of its study and its comprehension, this integrated review study was conducted based on the Broome method. This method is carried out in three stages of the search of texts, data evaluation and data analysis, so that in the search phase, the texts of postretrospective studies are examined in four stages in terms of inclusion criteria and after obtaining entry conditions The content of the study is evaluated and the data is analyzed at the end.

The present study is a systematic review study that was conducted in Persian and English by searching articles in search engines, scholar, Embase, ScienceDirect, PubMed, and Springer in the search engines, sites, and databases. Using the published articles in the last 24 years regarding the treatment of diabetic foot ulcer with an epidemiological approach. In the first phase, 36 articles were found. Of these, 12 articles related to the topic that was published in the last 24 years were reviewed.

The studies studied were written in English or Persian, access to their full text was possible, and entered the study, and unnamed, unannounced and non-academic studies were deleted.

To achieve relevant studies, a wide range of keywords including foot ulcer, diabetic patients, diabetic foot ulcer, epidemiologic approach , and diabetic foot ulcer treatment was used as a one-to-one search, combined with the method "And" and "OR".

\section{Result}

A foot ulcer is a major complication of diabetes mellitus with high morbidity, mortality, and associated costs, and therefore necessitates precautionary measures against diabetes mellitus (9). Therefore, due to the importance of this issue, this systematic review was conducted with the aim of determining the treatment of diabetic foot ulcer with an epidemiological approach.

In this clinical trial, conducted by Kargar et al. With the aim of determining the effect of platelet gel in the treatment of diabetic foot ulcers, 35 patients were treated with platelet gel and 35 subjects were treated with routine. Both groups received intravenous antibiotics Metronidazole and ceftriaxone), patients were evaluated for three weeks, there was no case of amputation in the platelet gel treatment group, and in the control group, foot ulcers in 6 patients resulted in limb amputation. (55). In another study, patients were randomly divided into two groups of light and laser lasers (placebo), 12-session laser therapy for 4 weeks with low-power laser gallium, aluminum. Low-level laser radiation is non-contagious on the surface of the lesions, changes in the area of lesions as the main outcome after treatment and up to the fourth month of

28 International Journal of Drug Research And Dental Science 
follow-up were evaluated (56). Also, in one study, the decrease in the mean area of the wound surface after treatment in the experimental group was higher than that in the control group, and as a result, when compressive suction treatment with the appropriate care of the foot ulcer was combined, it increased the repair of foot ulcer Diabetic (57).

Also, in a recent study, in the treatment of chronic wounds of grade 3 diabetes mellitus based on Wegener's classification, ultrasound waves of low frequency with standard care of the wound compared with standard care from the wound alone initially caused Accelerated recovery of chronic diabetic foot ulcers especially in the second and third months, while after 6 months of follow up, there was no significant difference in wound healing (58). In a study that aimed to report a case of diabetic foot ulcer treatment with topical administration of honey and olive oil, it was recommended that the patient first wash the wound completely with the serum and then place the mixture on a sterile gas and The wound was placed on the site and the dressing was replaced every 24 hours. After 5 days, granulation tissue was developed in the wound and the wound was completely recovered during one month (59). Also, in a study that aimed to report a case of diabetic foot ulcer treatment using heat-treated lambs in olive oil, the patient had a $1 \mathrm{~cm}$ incision at the interstitial wound, which, after referral, Penicillin powder and rinse with iodine were stopped and treated as daily dressing with topical mixture of heat-treated lambs in olive oil and animal oil, and the patient was advised to wash her wounds before dressing with serum and then Place the topical mixture on a sterile gas and place it on the wound and apply the bandage once every 12 hours. One week after starting treatment, new tissue was observed in the wound and the ulcer was completely closed within 2 weeks. The result is that the heat-treated wax in olive oil and animal oil is suitable for the treatment of diabetic foot ulcers (60).

In a study on 7 patients with type 2 diabetes with Wagner's 2 and 3 wounds, red radiation of $660 \mathrm{Nm}$ to the scar tissue and infrared $980 \mathrm{~nm}$ around the wound and red invertebrates with infrared lasers to some of the medical sites Needles for 5 to 10 days a day and then 2 times a week until complete wound healing repairs these wounds that have not recurred after 6 months (61). In a retrospective study on 48 patients before and after treatment, immersion ultrasound of necrotic tissue was debridled and the wound surface decreased and granulation tissue increased, and the rate of wound healing without scar formation and Slough was also higher (62). Another way to treat foot ulcers is to use an air cast diabetic walker boot to inflate the airbag inside the boot to reduce pressure on the skin, and also has a hard outer sheath and a deep heel that lowers the pressure (63). Another method that replaces the two-layer human skin that is used to treat intravenous ulcers and diabetic foot ulcers is Apligraft (64). In a clinical trial, tretinoin $0.5 \%$ solution for 10 minutes daily followed by iodine gel for 4 weeks was effective in wound healing than the control group (65). The results of this study showed that the effect of cold plasma radiation and endurance training did not significantly improve the wound healing in diabetic rats, but a period of endurance training and cold plasma also had a significant effect on wound healing in diabetic rats (66).

\section{Discussion}

Diabetes mellitus is considered as the most common disease caused by metabolic disorders and is a major global challenge. It is a disorder of insulin production and function disorder, which has led to an increase in uncontrolled outbreaks due to reduced physical activity and the consumption of unhealthy foods. (1-9). A foot ulcer is a major complication of diabetes mellitus with high morbidity, mortality, and associated costs, and therefore requires precautionary measures against diabetes mellitus (9). Therefore, due to the importance of this issue, this systematic review was conducted with the aim of determining the treatment of diabetic foot ulcer with an epidemiological approach.

In a study to determine the effect of platelet gel on the treatment of diabetic foot ulcers, it can generally be concluded that this study showed that platelet gel dressing was effective in the treatment of diabetic foot ulcers (55). Platelet growth factors are biologically active substances that accelerate the tissue repair

29 International Journal of Drug Research And Dental Science 
mechanism such as chemo-toxicance, cell proliferation, angiogenesis, and extracellular matrix sedimentation. Also, at the site of tissue damage, platelets release mitogenic and inflammatory materials that cover all aspects of tissue repair. In a study to determine the role of laser therapy in the treatment of diabetic foot ulcers on diabetic patients, showed that laser therapy can be effective in accelerating the healing of diabetic ulcers (56). The laser works with several mechanisms in these cases. In the first stage, vasodilatation increases the blood flow in the region, it also helps to destroy microorganisms in infectious wounds that strengthen the immune system. Another study also found that compressive suction treatment with the appropriate care of foot ulcers would increase the healing of diabetic foot ulcers. Therefore, it is recommended that patients with diabetic foot ulcer and chronic wound healing without recovery should use Vascular compression therapy for wound healing and organ preservation (57). In one study, with the aim of reporting a case of diabetic foot ulcer treatment with topical administration of honey and olive oil, after 5 days from the onset of treatment, granulation tissue was developed in the wound and the ulcer was completely improved within one month (59).

\section{Conclusion}

In the studies that have been studied, several therapeutic methods have been used to diabetic foot ulcers that have been effective. Considering that serious measures have been taken around the world to reduce complications of diabetes, including foot ulcers, but diabetes research centers in various countries and researchers working on diabetes must have serious plans to reduce complications of diabetic foot ulcers design and implement.

\section{Acknowledgment}

The authors are grateful to all those who cooperate with us.

\section{Conflict of interest:}

There are no conflicts of interest in this study.

\section{References}

1. Moslemirad M, Madmoli M, Madmoli Y, Niksefat M. Prevalence of type 1 and type 2 diabetes and its related factors in diabetic patients hospitalized in Khatam-ol-Anbia hospital in Shoushtar, 2014-15: A retrospective study. Journal of Research in Medical and Dental Science. 2018;6(3):421-6

2. Feyzi R, Madmoli M. Epidemiologic approach to quality of life in diabetic patients: a systematic review study. IJMBS [Internet].2019;3(5): 24-28. Available from: http://www.ijmbs.info/index.php/ijmbs/article/view/226

3. Feyzi R, Madmoli M. A systematic review with the epidemiological approach to the quality of life of cancer patients. IJMBS [Internet]. 2019;3(5): 18-23. Available from: http://www.ijmbs.info/index.php/ijmbs/article/view/235

4. Madmoli M, Madmoli M, Khodadadi M, Madmoli Y. The communication Between Diabetes and Alzheimer's Disease: a study on diabetic patients referring to health centers in three cities of Khuzestan province. International Research in Medical and Health Science [Internet]. 22Apr.2019 [cited 22Apr.2019];2(2):8-5. Available from: http://irmhs.com/index.php/irmhs/article/view/38.

5. Madmoli M, Madmoli M, Aliabad MA, Khodadadi M, Ahmadi FP. A systematic review on the impact of empowerment in improving self-care behaviors and some other factors in diabetic patients. International Journal of Health and Biological Sciences. 2019 Apr 13;2(1):11-6.

6. Madmoli M, Samsamipour M. The Most Important Methods for Reducing Chemotherapy-Induced Nausea and Vomiting in Cancer Patients: A Systematic Review Study. Int Health Res J. 2019;3(1):3-8. https://doi.org/10.26440/IHRJ/0301.04.521065

7. Madmoli M, Khodadadi M, Ahmadi FP, Niksefat M. A Systematic review on the impact of peer education on self-care behaviors of patients. International Journal of Health and Biological Sciences. 2019 Apr 13;2(1):1-5.

30 International Journal of Drug Research And Dental Science 
8. Madmoli M, Eilami O, Rezaie K, Aliabad MA, Moslemirad M. Diabetes and the risk of suffering cardiovascular Diseases: A two-year retrospective study. International Journal of Ecosystems and Ecology Science (IJEES). 2018 Jun;8(3): 649-56.

9. Madmoli M Rostami F, Mirsami Yazdi N, Mosavi A, Baraz Sh. Evaluation of Prevalence of Diabetic Foot Ulcer and Its Related Factors in Diabetic Patients Admitted to KHatam-ol-Anbia Hospital in Shoushtar During 2015-2016: A Retrospective Study. International Journal of Ecosystems and Ecology Science (IJEES). 2018 June; 8 (3): 545-52.

10. Rostami F, Madmoli M, Mirsami Yazdi N, Baraz Sh. Evaluation of The Prevalence of Lower Limb Amputation and Its Related Factors in Diabetic Patients Admitted to KHatam-ol-Anbia Hospital in Shoushtar During The 2015-2016: A Retrospective Study. International Journal of Ecosystems and Ecology Science (IJEES). 2018 June; 8 (3): 553-60.

11. Madmoli M, Madmoli Y, Mobarez F, Taqvaeinasab H, Darabiyan P, Rafi A. Drugs abuse and increase in referral to hospital to prevent recurrence of diabetic foot ulcer infection. International Journal of Ayurvedic Medicine. 2019 Apr 3;10(1):89-94.

12. Raisifar Z, Afshar Nia A, Madmoli M, Madmoli Y. The Relationship Between Using Insulin and Suffering Alzheimer's Disease in Patients with Diabetes: A Two-Year Study. International Journal of Ecosystems and Ecology Science (IJEES). 2018 June; 8 (3): 623-28.

13. Madmoli M, Izadi M, Madmoli M. A Systematic Review Study on Interventions to Reduce Nausea and Vomiting Caused by Chemotherapy. Journal of Biotechnology and Bioengineering. 2019; 3(1):14-20.

14. Madmoli M, Izadi M, Khodadadi M, Khodadadi M, Papi Ahmadi F, Abbaszade Aliabad M.A Systematic Review Study on the Effect of Multimedia Training on Self-Care Behaviors and Quality of Life in Diabetic Patients. Journal of Genetics and Genetic Engineering, 2019; 2(4): 1-6.

15. Madmoli M, Izadi M, Madmoli M, Khodadadi M, Ahmadi FP, Aliabad MA. A Systematic Review Study of the Most Important Complications of Chemotherapy in Cancer Patients. Journal of Biotechnology and Bioengineering. 2019; 3(1): 2-7

16. Raisifar Z, Afshar Nia A, Maghamesi Moarrefi H, Madmoli M. Evaluation of Gi Bleeding Prevalence and Its Related Factors in Diabetic Patients Hospitalized in KHatam-ol-Anbia Hospital During 2015-16: A Retrospective Study. International Journal of Ecosystems and Ecology Science (IJEES). 2018 June; 8 (3): 609-14.

17. Madmoli M, Kord Z, Bandani A, Sedighi N, Rezaei Shandiz M, Darabiyan P, AfsharNia A. Epidemiological and clinical study of patients with Alzheimer's in Five Cities of Khuzestan Province in 2016-2018. Medical Science, 2019; 23(95), 1-5

18. Mashali H, Toleideh F, Rahmani R, Darabiyan P, Madmoli M. The predictive role of Hyperlipidemia in the incidence of ACS in patients referring to Shahidzadeh Hospital in Behbahan in 2016 -2017. Medical Science, 2018; 22(94), 566-570

19. Madmoli M, Modheji Y, Rafi A, Feyzi R, Darabiyan P, AfsharNia A. Diabetes and its predictive role in the incidence of Alzheimer's disease. Medical Science, 2019; 23(95), 30-34

20. Madmoli M, Shaidaei MF, Rohani A, Madmoli Y, Khodadadi M. Some predisposing factors and affecting cancer under the age of 35: A 6-year study on 2721 cancer patients. International Journal of Ayurvedic Medicine. 2019 Apr 3;10(1):62-7.

21. Rafiee E, Khaledi M, Madmoli M, Zafari M, Lotfizadeh M. The Correlation between Blood Pressure and BMI in Students of Shahrekord University of Medical Sciences in 2013-14. International Journal of Ayurvedic Medicine. 2019 Apr 3;10(1):113-7.

22. Madmoli M, Saeidilandi M, Latifinasab R, Fatehimoghaddam S, Mobarez F, Darabiyan P. Hypertension and Risk of Acute Coronary Syndrome (ACS) in Patients with ACS: A Study on 926 patients with ACS. International Journal of Ayurvedic Medicine. 2019 Apr 3;10(1):22-6.

31 International Journal of Drug Research And Dental Science 
23. Madmoli M, Madmoli Y, Taqvaeinasab H, Khodadadi M, Darabiyan P, Rafi A. Some influential factors on the severity of diabetic foot ulcers and Predisposing of limb amputation: A 7-year study on diabetic patients. International Journal of Ayurvedic Medicine. 2019 Apr 3;10(1):75-81.

24. Madmoli M, Abbaszade Aliabad M, Madmoli M, Khodadadi M, Papi Ahmadi F. The Effect of Some Factors on Self-Care in Diabetic Patients: A Systematic Review. Journal of Genetics and Genetic Engineering. 2019; 3(1): 21-25

25. Madmoli M. A Systematic Review Study on the Changer Factors of the Quality of life in Cancer Patients. Int. Res. Med. Health Sci., 2019; 2(1):8-15.

26. Madmoli M. A systematic Review Study on the Results of Empowerment-Based Interventions in Diabetic Patients. Int. Res. Med. Health Sci., 2019; 2(1):1-7.

27. Madmoli M, Fallah bagher shaidaei M, Rohani A, Darabiyan P, Mobarez F. The correlation between alcohol consumption and reducing the age of cancer incidence in patients with this disease. Medical Science, 2019, 23(95), 48-53

28. Madmoli M, Mahmoudi Dehcheshmeh Z, Rafi A, Zahra Kord, Fariba Mobarez, Pouriya Darabiyan. The rate of some complications and risk factors of diabetes in diabetic patients: Study on cases of 3218 diabetic patients. Medical Science, 2019; 23(95), 63-68

29. Mostafa Madmoli, Mehran Yarbig, Negin Sedighi, Pouriya Darabiyan, Fariba Mobarez. Communication between body mass index and the risk of obesity-related cancer: A 5-year study on patients with cancer. Medical Science, 2019; 23(95), 69-74

30. Madmoli M. Clinical and Laboratory Finding Children with Leukemia: a Systematic Review. International Journal of Research Studies in Science, Engineering, and Technology, vol. 5, no. 10, pp . 2018; 1-6

31. Madmoli M. Evaluation of Chemotherapy Complications in Patients with Cancer: A Systematic Review. International Journal of Research Studies in Science, Engineering, and Technology, vol. 5, no. 12, 2018; 59-64.

32. Madmoli M, Madmoli Y, Khodadadi M, Samsamipour M. Study of Some Effective Treatments for Accelerating Diabetic Foot Ulcer Healing: A Systematic Review. International Journal of Research Studies in Science, Engineering, and Technology. 6(2), 2019; 34-39

33. Madmoli M, Madmoli Y, Khodadadi M, Samsamipour M. Factors Affecting the Level of Glycosylated Hemoglobin in Patients with Diabetes: A Systematic Review. Annals of Microbiology and Infectious Diseases 2(1), 2019; 43-47.

34. Madmoli, M. A Systematic Review Study on the Communication between Diabetes and Cancer: The Serious Risk of Cancer in Diabetic Patients. International Research in Medical and Health Science. 2019; 2(2), 1-7.

35. Madmoli M. Quality of Life in Patients with Cancer and Some Factors Affecting it: A Systematic Review. International Journal of Research Studies in Science, Engineering, and Technology. 6(1), 2019; $1-7$

36. Madmoli M, Madmoli Y, Khodadadi M, Samsamipour M. Study of Some Effective Treatments for Accelerating Diabetic Foot Ulcer Healing: A Systematic Review. International Journal of Research Studies in Science, Engineering and Technology, 6(2), 2019; 34-39

37. Madmoli M, Madmoli Y, Khodadadi M, Samsamipour M. Factors Affecting the Level of Glycosylated Hemoglobin in Patients with Diabetes: A Systematic Review. Annals of Microbiology and Infectious Diseases 2(1), 2019; 43-47. 
38. Madmoli M, Madmoli Y, Khodadadi M, Samsamipour M. Some Factors Affecting Quality of Life in Patients with Diabetes: A Systematic Review. Annals of Microbiology and Infectious Diseases, 2(1), 2019; 26-30.

39. Gheisari Z, Beiranvand R, Karimi A, Ghalavandi S, Soleymani A, Madmoli M, Bavarsad AH. Relationship between Occupational Stress and Cardiovascular Risk Factors Determination: A Casecontrol Study. Journal of Research in Medical and Dental Science. 2018 May 17;6(3):287-93.

40. Madmoli M, Nikpay S. An Investigation of the Relationship between Spiritual Health and Depression, Anxiety, and Stress among Students of Ilam University of Medical Sciences. Journal of Research in Medical and Dental Science. 2018 May 17;6(3):294-300.

41. Adavi A, Hamid N, Attari Y, Madmoli Y, Madmoli M. Study of the Effect of Problem-Solving Skills Training on Creativity and Assertiveness Among High School Students. Iranian Journal of Nursing Research. 2016 Dec 15;11(5):48-55.

42. Madmoli M, Madmoli Y, Bigdeli Shamloo MB, Etebari A, Mahmoodi Kouhi A, Azami M. The Relationship Between Depression and Religiousness in Female High School Students of Masjed Soleyman in 2015. Journal of Pediatric Nursing. 2017 Jun 15;3(4):15-22.

43. Roughani A, Madmoli M, Raisifar Z, Kikhavani S, Yasemi M, Azami M, Sharifi Z. The prevalence of behavioral disorders and its related factors in elementary school children in Ilam City in 2011-2012. Indian Journal of Forensic Medicine \& Toxicology. 2018;12(4):165-9.

44. Madmoli Y, Madmoli M, Qashqaei Nezhad N, Bosak S. Prevalence of depression and associated factors in adolescents of Masjed-Soleyman. Iranian Journal of Pediatric Nursing. 2016; 2(4):22-26.

45. Madmoli Y, Aslani A, Ahmadi Y, Mousavi M, Mashalchi H, Niksefat M, Madmoli M. Study Habits and Related Factors in Students of Nursing and Midwifery College of Dezful University of Medical Sciences in 2015. Iranian Journal of Nursing Research (IJNR). 2017.

46. Madmoli Y, Akhaghi Dezfuli SM, Beiranvand R, Saberi Pour B, Azami M, Madmoli M. An epidemiological and clinical survey of patients with $\beta$-thalassemia in dezful in 2015. Iranian Journal of Epidemiology. 2017;13(2):145-52.

47. Madmoli Y, Salimi M, Madmoli M, Davoodi N, Rostami F, Heidari-Soureshjani R, Yousefi N. The relationship between spiritual well-being and family cohesion in patients with thalassemia major, J Res Med Dent Sci, 2019, 7(2):203-209.

48. Madmoli Y, Salimi M, Madmoli M, Maraghi E, Pelarak F, Korkini N, Mashalchi H.The effect of orem self-care model on health-related quality of life of patients with thalassemia major, J Res Med Dent Sci, 2019, 7(2): 170-176.

49. Madmoli Y, Madmoli M, Davoodi N, Niksefat M, Saberipour B, Darabiyan P, Yousefi N. Health-related quality of life in patients with a $\beta$-thalassemia major in northern Khuzestan Province, southwest of Iran in 2015-2016, J Res Med Dent Sci, 2019, 7(2): 164-169.

50. Madmoli Y, Akhaghi Dezfuli SM, Adavi A, Maraaghi E, Heidari Soureshjani R, Madmoli M. The Effect of Orem Self-Care on Mental Health of Patients with Thalassemia Major. Journal of Clinical Nursing and Midwifery. 2018 Jul 1;7(2):108-15.

51. Madmoli M, Madmoli Y, Rahmati P, Adavi A, Yousefi N, Gheisari Z, Abbaszade Aliabad M. Quality of Life and Some Related Factors in Patients with Beta Thalassemia Major in Southwest Iran. Journal of Client-centered Nursing Care (JCCNC. 2017;3(2).

52. Madmoli Y, Beiranvand R, Korkini N, Mashalchi H, Karimi H. [Comparison of health-related quality of life in beta-thalassemia major and healthy people in Dezful in 2015. Iran J Nurs Res. 2016; 11(1):916. 
53. Madmoli Y, Akhaghi Dezfuli SM, Beiranvand R, Saberi Pour B, Azami M, Madmoli M. [An epidemiological and clinical survey of patients with $\beta$-thalassemia in dezful in 2015. Iran J Epidemiol. 2017; 13(2):145-52.

54. Maraghi E, Adavi A, Madmoli Y, Heidari-Soureshjani R, Madmoli M. The Effect of Orem Self-Care on Mental Health of Patients with Thalassemia Major. Journal of Clinical Nursing and Midwifery. 2018;4(3).

55. Kargar S, Javadzadeh Shahshahani H, Tabkhi N. The effect of platelet gel on the treatment of diabetic foot ulcer. Sci J Iran Blood Transfus Organ. 2010; 6 (4) :283-291. URL: http://bloodjournal.ir/article-1-368-fa.html

56. Atayi Feshtemi L, Esmaeili Javid Gh, Kaviani A, Larijani B, Razavi L, Salami M. Low-Level Laser Therapy in Treatment of Diabetic Foot Ulcers: Double-Blind Controlled Clinical Trial. Lasers in medicine. 1387; 5 (3).

57. Moodi H, Akbari A, Ghiasi F, Mahmoudzadeh H, Heidari Z, Rashidi H. The Effect of VacuumCompression Therapy (VCT) on the Diabetic Foot Ulcer Healing. zumsj. 2006; 14 (57) :15-22. URL: http://zums.ac.ir/journal/article-1-128-fa.html

58. Amini S, Rezaei Hemami M, Anabestani Z, Shayegan Mehran Z, Ardeshirralijani M B, Mohajeri Tehrani M R. The effect of low-frequency ultrasound waves on diabetic foot ulcers with osteomyelitis in a randomized clinical trial. 2011; 543-552.

59. Zahmatkash M, Rashidi M. Treatment of Diabetic Leg Ulcer by Local Application of Honey and Olive Oil Mixture. JMP. 2009; 4 (29) :36-40. URL: http://imp.ir/article-1-381-fa.html

60. KHADEM HH, KOUSHAN Y, ASGHARZADEH A. Treatment of diabetic foot ulcer with propolis and olive oil: a case report. 2012; 35-38.

61. N. Kazemi-Khoo. Successful treatment of diabetic foot ulcers with low-level laser therapy. The foot, December 2006; 16(4): 184-187.

62. Jaimee Haan PT, Sharon Lucich PT. A Retrospective Analysis of Acoustic Pressure Wound Therapy: Effects on the Healing Progression of Chronic Wounds. J Am Col Certif Wound Spec 2009; 1(1): 28-34.

63. James C. Stanley and Andrew M. Collier. The diabetic foot and ankle. Orthopaedics and Trauma, 2009; 23 (1): 61-68.

64. RonaldsWarren.http://www.fda.gov/MedicalDevices/ProductsandMedicalProcedures/DeviceAppro valsandClearances/Recently- ApprovedDevices/ucm085085.html

65. McCulloch D, Asla R. Management of diabetic foot lesions. Up to Date Ver 18.1 (updated 31 Jan 2010).

66. Aryandoost S, Nazarali P, Fathi R, Sohbatzadeh F. THE EFFECT OF ENDURANCE EXERCISE ON WOUND HEALING OF DIABETIC RATS BY COLD PLASMA. Iranian Journal of Diabetes and Metabolism. 2017; 16 (2) :95-102. URL: $\underline{\text { http://ijdld.tums.ac.ir/article-1-5495-fa.html }}$

How to cite this Article: Zahra Shiri¹, Zeinab Zarinzade', Mohammad Khalilikia², Ahmad Aslani², Hadi Kianirad 2*, Yones Adish2, Treatment of Diabetic Foot Ulcer With An Epidemiological Approach: A systematic Review Study, . Int. J. Drug Res. Dental Sci., 2019; 1(1): 27-34.

Source of Support: Nil, Conflict of Interest: None declared. 